\title{
Жидкокристаллические устройства для модуляции терагерцового излучения
}

\author{
В. Беляев, д. т.н. ${ }^{1}$
}

УДК 532.783 | ВАК 05.27 .01

\begin{abstract}
В последние годы особенно актуальными стали задачи, связанные с использованием терагерцового диапазона спектра для устройств обработки информации, оптоэлектроники, сВЧ-электроники, медицины. Возникает необходимость формирования пучков излучения и даже двумерных изображений в этом диапазоне, как статических, так и в виде динамической последовательности.
\end{abstract}

1 ля видимого и инфракрасного диапазонов проблемы формирования пучков излучения и двумерных изображений как статических, так и в виде динамической последовательности решены [1, 2], а для ТГц-диапазона решение таких задач с возможностью промышленного выпуска приборов пока отсутствует. При этом стоит отметить, что устройства ТГцдиапазона для обнаружения скрытых объектов с выводом изображений на визуальные дисплеи известны достаточно хорошо [3-5].

В настоящее время существует несколько подходов в создании модуляторов ТГц-излучения. Кним относится оптическая и электронная модуляция в полупроводниках и метаматериалах [6], электронная модуляция в графене [7], термальная модуляция [8], а также модуляция фотонными кристаллами [8]. К основным недостаткам вышесказанных методов в той или иной мере можно отнести низкую глубину модуляции, резонансный характер модуляции (модуляция в узкой полосе пропускания), а также низкую скорость модуляции. Исходя из этого, актуальной задачей является поиск новых оптоэлектронных модуляторов терагерцового излучения, имеющих количественное преимущество в рабочих характеристиках перед имеющимися на данный момент устройствами такого типа.

жидкие кристаллы (ЖК) являются хорошим кандидатом на роль управляющих материалов ввиду высокой величины двойного

Московский государственный областной университет (Мгоу), профессор, главный научный сотрудник, заведующий кафедрой теоретической физики; инженерная академия Российского университета дружбы народов, профессор департамента механики и мехатроники. лучепреломления как в видимом, так и в микроволновом диапазонах электромагнитного спектра, малого рабочего напряжения, малых времен электрооптического отклика, широкого диапазона рабочих температур [9]. Тем не менее, у таких ЖК-модуляторов есть ряд недостатков, таких как необходимость использования толстых слоев ЖК ввиду большой длины волны в ТГц-режиме, что приводит к высоким рабочим напряжениям и большому времени отклика [10]. Эту проблему можно преодолеть за счет использования ЖК с большой величиной двулучепреломления, методов модуляции, в частности, дифракционных методов, а также за счет изменения условий применения устройства, например вариацией угла падения ТГц-излучения на модулятор.

В современной литературе есть ряд примеров разработки перестраиваемых ЖК-устройств ТГц-диапазона $[9,10]$.

жК-ячейка в работе [9] имеет структуру, изображенную на рис. 1. На стеклянную подложку 1 наносится графеновая пленка 2, которая одновременно выполняет роль как прозрачного электрода, так и поляризатора. Для равномерного ориентирования ЖК необходим

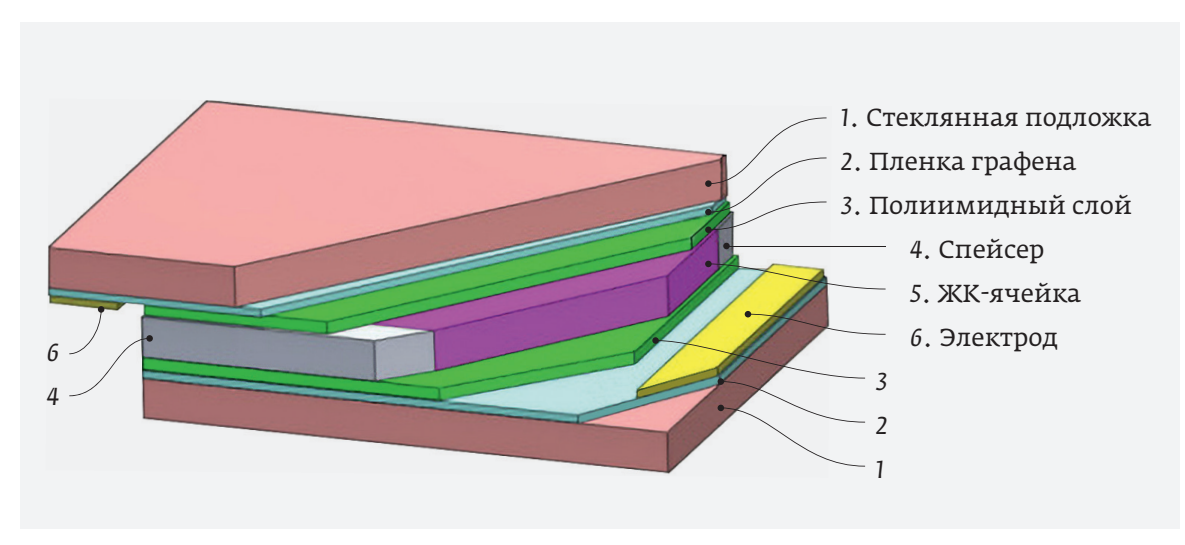

Рис. 1. Схематическая диаграмма многослойной жК-ячейки, используемой в качестве фазовращателя в работе [9] 
полиимидный слой 3, а для получения заданной толщины ЖК образца 5 используют спейсеры 4. В отсутствие электрического поля ориентация ЖК в ячейке - планарная, при приложении электрического поля в ячейке формируется гомеотропная ориентация.

К недостаткам использования графена можно отнести сложность создания графенового слоя с минимальным количеством дефектов в узлах решетки, что существенно снижает его электрофизические свойства и дает большой разброс в характеристиках получаемых ячеек. Технология применения графена, конечно, перспективна, но ее промышленное использование возможно только через достаточно длительное время. Поэтому

$$
\begin{aligned}
& \text { (l) }
\end{aligned}
$$

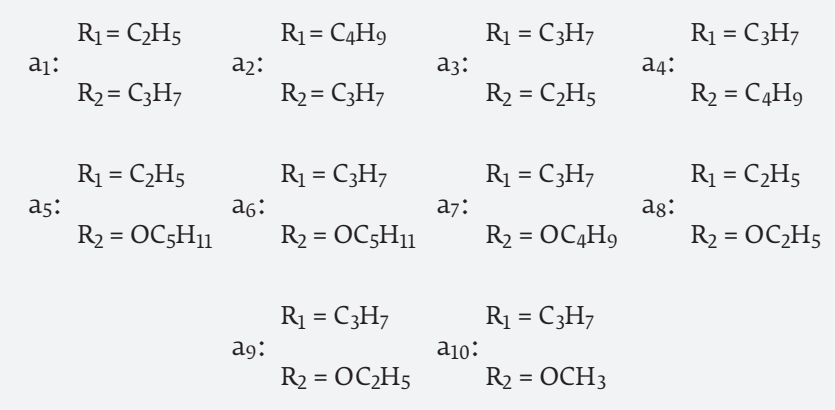

a)

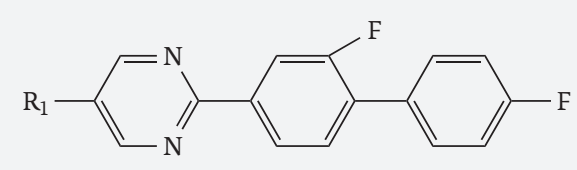

$\mathrm{b}_{1}: \mathrm{R}=\mathrm{C}_{3} \mathrm{H}_{7} \quad \mathrm{~b}_{2}: \mathrm{R}=\mathrm{C}_{5} \mathrm{H}_{11}$

б)

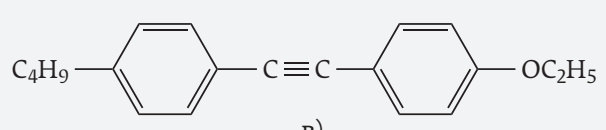

в)

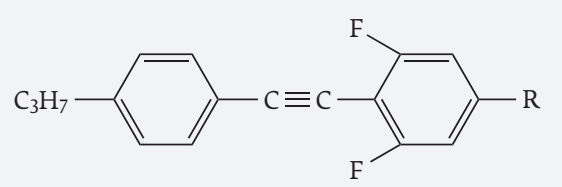

$\mathrm{d}_{1}: \mathrm{R}=\mathrm{C}_{2} \mathrm{H}_{5} \quad \mathrm{~d}_{2}: \mathrm{R}=\mathrm{OCH}_{3}$

г)

Рис. 2. Молекулярная структура компонент НЖК-смеси NJU-LDn-4 следует использовать доступные, производимые в настоящее время, материалы и технологии с адаптацией ихктГц-диапазону.

Разработана нематическая ЖК-смесь (НЖК-смесь) NJU-LDn-4 с большой величиной двойного лучепреломления, состоящая из нескольких фторированных производных фенилтолана [10]. Компоненты данной смеси представлены на рис. 2.

Основным компонентом является смесь (а) из 10 фторированных соединений бифенил-толанов, составляющая 76\% массовой доли. Массовая доля отдельных компонентов этой смеси варьируется от 2 до 10\%. Компонент (б) с пиримидиновым кольцом составляет 12\% массовой доли, а компоненты (в) и (г) соответственно 5 и $7 \%$.

Зависимость показателя преломления обыкновенного $\left(n_{0}\right)$ и необыкновенного $\left(n_{e}\right)$ лучей, а также двулучепреломления $(\Delta n)$ в диапазоне 0,4-1,6 ТГц представлена на рис. 3. Среднее значение двулучепреломления в данном диапазоне равно 0,306, а максимальное - 0,314 при 1,6 ТГц. Физические свойства ЖК-материалов на основе толанов и их зависимость от молекулярного строения описаны в [11].

На основе данной НЖК-смеси с высоким двулучепреломлением разработана специальная ячейка, являющаяся перестраиваемым фазовращателем ТГц-излучения. Принципиальная схема данной ячейки изображена на рис. 4. Ячейка состоит из двух подложек из кварцевого стекла с зазором в 250 мкм, заполненным ЖК. На внутренние поверхности подложек наносятся решетка из золота и полиимидный ориентирующий слой. Направления решеток на двух подложках располагают параллельно друг другу. Направление натирания полиимидных слоев, в свою очередь, задают перпендикулярно

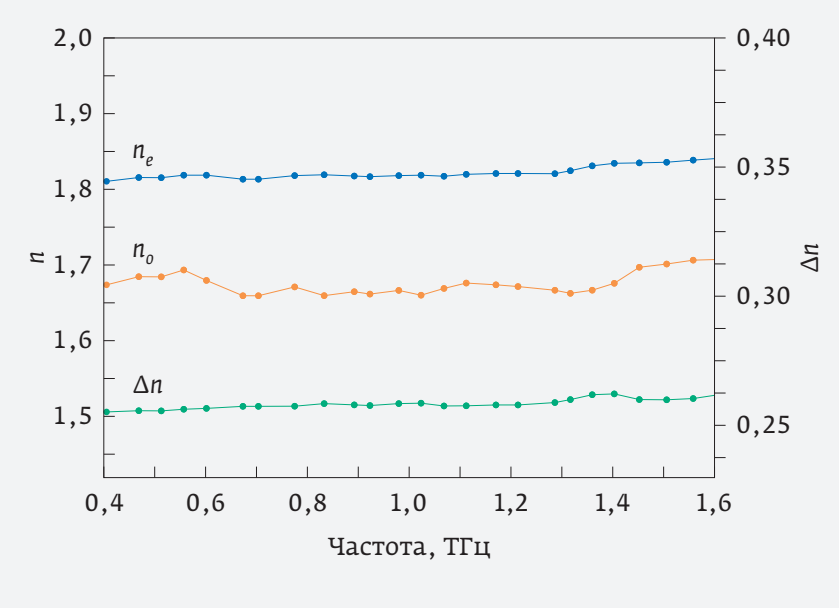

Рис. 3. Частотная зависимость двулучепреломления HжK-смеси NJU-LDn-4 в диапазоне 0,4-1,6 ТГц 


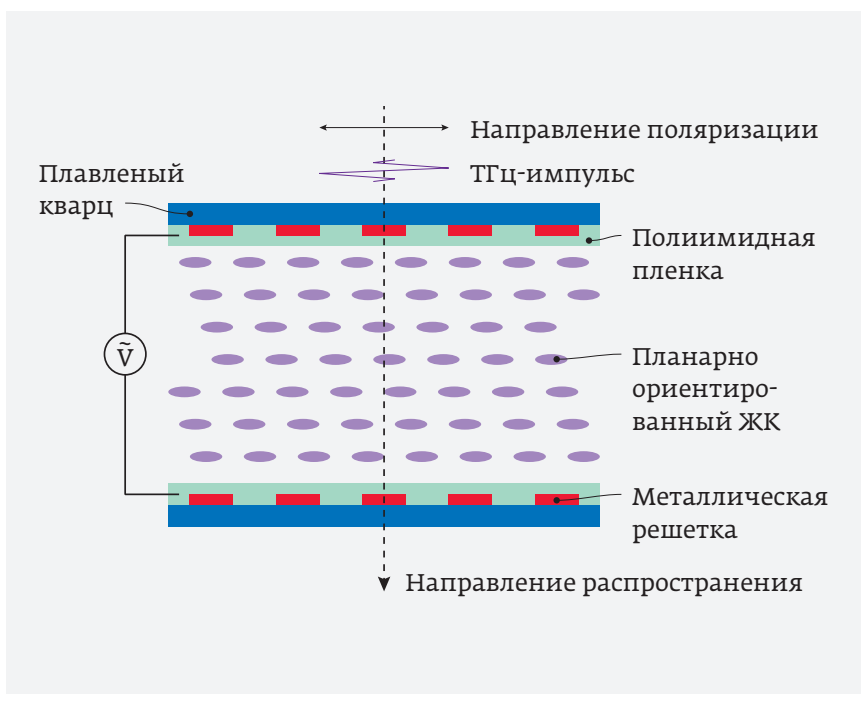

Рис. 4. Схематическое изображение ЖК поляризационного перестраиваемого фазовращателя в ТГц-диапазоне [10]

золотым полоскам, что обеспечивает планарную ориентацию. Период решетки составляет 20 мкм, а ширина 10 мкм, длина волны исследуемых ТГц-волн в таком случае составляет гораздо бо́льшую величину. Благодаря этому данная ячейка способна пропускать поперечные магнитные волны, в то время как поперечные электрические волны будут отражаться. Поэтому решетка из золота в данной ячейке выполняет двойную роль: прозрачных электродов и встроенных поляризаторов.

Перспективность использования композиционных материалов типа пористая полимерная мембрана - жидкий кристалл (ПМЖК) для модуляции видимого и ТГц-излучения показана в работах [12]. В этой системе достаточно длинный путь распространения волны терагерцовой частоты, позволяющий получить фазовый набег до четверти длины волны, при малом (десятки микрометров) размере переключаемого электрооптического ЖК-элемента в пористой матрице.

Переключаемые фазосдвигатели на основе как обычных ЖК, таки ЖК, диспергированных в полимере [1], описаны в $[13,14]$.

Таким образом, показана возможность реализации модуляции ТГц-излучения и создания изображений в этом диапазоне с достаточно высоким пространственным разрешением и высокой скоростью модуляции на основе жидкокристаллических элементов.

Работа частично поддержана Российским фондом фундаментальных исследований, грант № 19-07-00602_а.

\section{ЛИТЕРАТУРА}

1. Беляев В. В. Жидкокристаллические дисплеи. Технологии настоящего и будущего. Часть 2. Новые технологии и области применения ЖК-дисплеев // ЭЛЕКТРОНИКА: Наука, Технология, Бизнес. 2015. № 10. С. 124-131.

2. Грузевич ю. Оптико-электронные приборы ночного видения. - Физматлит, 2014

3. Sheen D. M., McMakin D. L., Hall T. E. Three-dimentional millimeter-wave imaging for concealed weapon detection // IEEE Transactions on Microwave Theory and Technique. 2001. V. 49. № 9. P. 1581-1592.

4. Rebeiz G. M., Kasilingam D. P., Guo Y., Stimson P. A., Rutledge D. B. Monolithic millimeter-wave twodimensional horn imaging arrays // IEEE Transactions on Antennas and Propogation. 1990. V. 38. № 9. Р. 1473-1482.

5. Шашкин В. И., Белов Ю.И., Волков П. В., Горюнов А. В., Закамов В.Р., Илларионов И. А. Экспериментальное исследование матрицы детекторов системы радиовидения 3-mm диапазона длин волн // Письма в Журнал технической физики. 2013. Т. 39. Вып. 12. C. 44-49.

6. Karl N., Reichel K., Chen H.-T., Taylor A. J., Brener I., Benz A., Reno J.L., Mendis R., Mittleman D. M. An electrically driven terahertz metamaterial diffractive modulator with more than $20 \mathrm{~dB}$ of dynamic range // Applied Physics Letters. 2014. V. 104. IS. 9. P. 091115.

7. Sensale Rodriguez B., Yan R., Liu L., Jena D., Xing H. G. Graphene for Reconfigurable THz Optoelectronics // Proceedings of the IEEE. 2013. V. 101. Is. 7. P. 1705-1716.

8. Rahm M., Li J.-S., Padilla W.J. THz Wave Modulators: A Brief Review on Different Modulation Techniques // Journal of infrared, millimeter and terahertz waves. 2013. V. 34. Is. 1. P. 1-27.

9. Wu Y., Ruan X., Chen C.-H., Shin Y.J., Lee Y., Niu J., Liu J., Chen Y., Yang K.-L., Zhang X., Ahn J.-H., Yang H. Graphene / liquid crystal based terahertz phase shifters // Optics Express. 2013. V. 21. Is. 18. P. 21395-21402.

10. Lu Y.-Q., Hu W., Liang X. THz Devices based on High Birefringence Liquid Crystals // SID Symposium Digest of Technical Papers. 2014. V. 45. Is. 1. P. 491-494.

11. Беляев В.В. Определение анизотропии локального поля и параметра порядка 4-алкил-4'-алкокситоланов по рефрактометрическим данным // Журнал физической химии. 2001. Т. 75. Вып. 6. С. 1088-1094.

12. Pasechnik S., Chopik A., Shmeliova D., Drovnikov E., Semerenko D., Dubtsov A., Zhang W., Chigrinov V. Electro-kinetic phenomena in porous PET films filled with liquid crystals // Liq. Cryst. 2015. 42. P. 1537-1542.

13. Ito R., Nose T., Ozaki M., Takeya K., Tonouchi M. THz Wave Transmission Properties of LC Composite Membrane Films // Mol. Cryst. Liq. Cryst. 2010. 516. P. 144-151

14. Ito R., Kumagai T., Yoshida H., Takeya K., Ozaki M., Tonouch M., Nose T. THz Nematic Liquid Crystal Devices Using Stacked Membrane Film Layers // Mol. Cryst. Liq. Cryst. 2011. 543. 77 / [843]-84/[850] 


\section{ОБОРУДОВАНИЕ ДЛЯ МИКРОЭЛЕКТРОНИКИ}

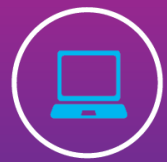

ПРОЕКТИРОВАНИЕ

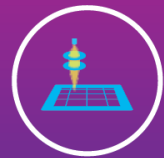

ГЕНЕРАЦИЯ ИЗОБРАЖЕНИЙ

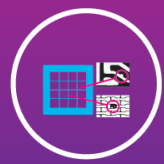

КОНТРОЛЬ ФОТОШАБЛОНОВ

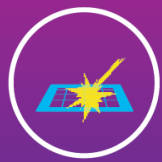

PEMOHT ФОТОШАБЛОНОВ

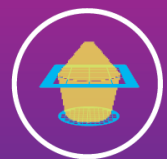

ФОТОЛИТОГРАФИЯ

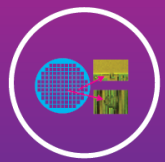

КОНТРОЛЬ ПЛАСТИН

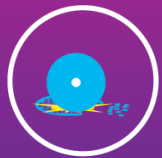

СБОРКА ИЗДЕЛИЙ МИКРОЭЛЕКТРОНИКИ
БЕЗМАСОЧНАЯ ЛИТОГРАФИЯ

- Многоканальные лазерные генераторы изображений

- Проектная норма 0.35, $0.6 \mu \mathrm{m}$

- Высокая точность совмещения

- $\oslash 200,150,100$ мM

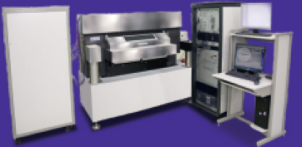

ГЕНЕРАТОРЫ ИЗОБРАЖЕНИЙ

- Диапазон UV, DUV

- Проектная норма 90,130 нм

- 16/32-лучевая архитектура

- Фазосдвигающие шаблоны

- Быстрая переналадка

пластина - шаблон

КОНТРОЛЬ ФОТОШАБЛОНОВ

- Проектная норма 90,130, 250 нм

- Твердотельный лазер

- Контроль методом D2DB, D2D

- Высокая производительность

- Контроль неплоскостности

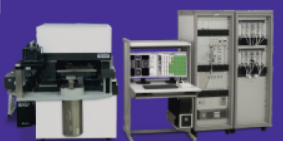

РЕМОНТ ФОТОШАБЛОНОВ

- Фемтосекундный лазер
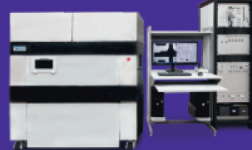

- 0.15/ 0.3/ 0.5 um min элемент

- Размер шаблона до 9"х9"

- Ремонт копированием

- Ремонт через пелликл

- Прозрачные / непрозрачные дефекты

КОНТАКТНАЯ ЛИТОГРАФИЯ

- Ручная и автоматизированная загрузка

- Двусторонняя литография

- Высокая точность совмещения

- Низкий уровень генерации дефектов

- Высокая энергоэффективность

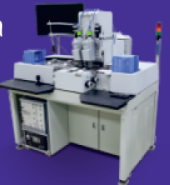

СТЕППЕРЬ

- Проектная норма 0.35, 0.8 um

- Автоматический масштаб

- Двустороннее совмещение

- $\oslash 200,150,100$ мм

- Твердотельный источник света
КОНТРОЛЬ ТОПОЛОгИИ

- Контроль привносимых дефектов

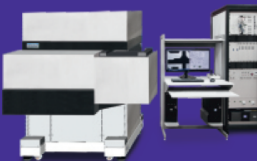

пластин без топологии

- Автоматический микро и макро контроль дефектов пластин

с топологией

- Высокая производительность

АВТОМАТИЧЕСКИЙ КОНТРОЛЬ И ИЗМЕРЕНИЕ

- Контроль микроразмеров

- Контроль неплоскостности

- Контроль координат

- Контроль толщин

- Контроль рассовмещения

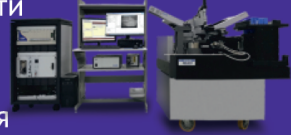

- Единое таможенное пространство

- 58 лет опыта в разработке и производстве прецизионного оптико-механического и сборочного оборудования

- Высокий уровень применяемых технологий и современного оборудования

- Полный цикл разработки и производства, высококвалифицированный персонал

- Высокое качество изделий подтверждено национальными и международными стандартами

- Возможность комплексной поставки оборудования, адаптированного для Российского рынка, программного обеспечения для поддержки процессов изготовления фотошаблонов и 3D-моделирования для фотолитографии компании GenISys (Германия)

Республика Беларусь

220033, г. Минск

Партизанский пр-т, 2 office@kbtem-omo.by

kbtem.omo@gmail.com

www.planar.by

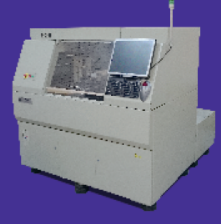

\section{ЗОНДОВЫЙ коНТРОЛЬ}

Ручное / полуавтоматическое / автоматическое оборудование

РАЗДЕЛЕНИЕ ПЛАСТИН И ПОЛЛОЖЕК

одной установке

кВт мощность электрошпинделя

плуавтоматическое

автоматическое оборудование

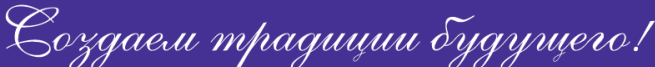

